\title{
Prevalence and molecular studies on Echinococcus equinus isolated from necropsied donkeys
}

\author{
A.Y. Desouky ${ }^{1}$, N.M. Helmy ${ }^{2}$, Sh.S. Sorour ${ }^{1}$ and M.M. Amer ${ }^{2} *$ \\ ${ }^{1}$ Parasitology Department, Faculty of Veterinary Medicine, Kafr El-Sheikh University, Kafr El-Sheikh 33516, Egypt \\ ${ }^{2}$ Biotechnology Department, Animal Health Research Institute, Dokki 12618, Egypt \\ "Corresponding Author: mooaz.amer@gmail.com
}

(Received August 14, 2017; Accepted September 18, 2017)

\begin{abstract}
In the present study, forty donkeys of different ages and sexes at Giza Zoo, Egypt were investigated between October 2015 and September 2016 for the presence of hydatidosis disease. Hydatid cysts were detected in the livers of 10\% of the examined donkeys and these cysts had a fertility rate $100 \%$. Female donkeys were infected with cysts more than males and all infected donkeys were old aged with no cases of infection were detected in young or adult donkeys. Using molecular tools, the DNA extracted from cysts that had been isolated was subjected to PCR amplification, using synthesized oligonucleotide primers, and these were constructed to target the $299 \mathrm{bp}$ within the (ND2) gene, which is considered to be specific for the Echinococcus equinus genotype. The sequenced PCR products showed homology to E.equinus (G4 or horse strain genotype). These results can be used in future to pursue the epidemiological status of the causative strain of hydatidosis in equines at the study area.
\end{abstract}

Keywords: hydatidosis, PCR, horse strain, equines

Available online at http://www.vetmedmosul.org/ijvs

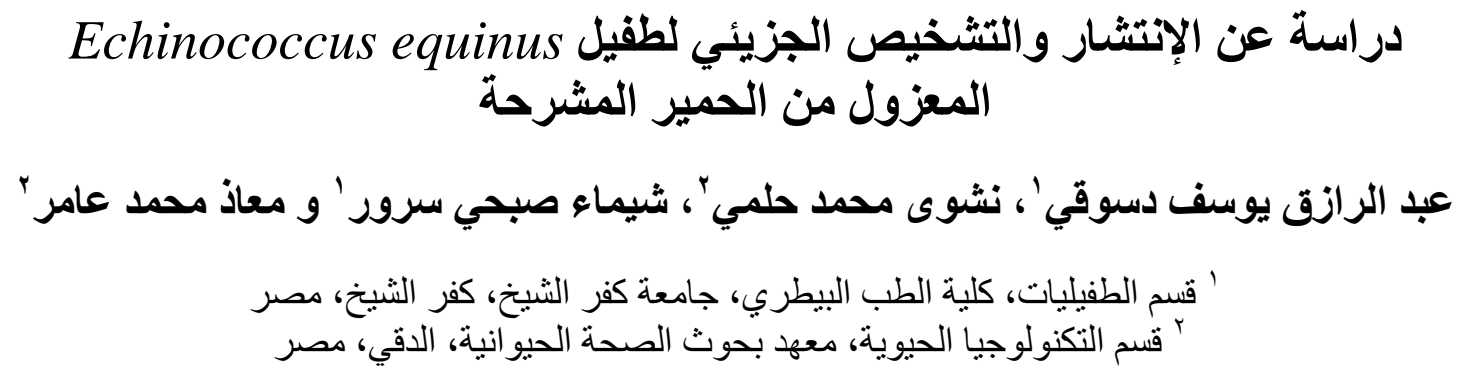

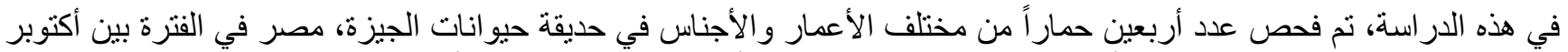

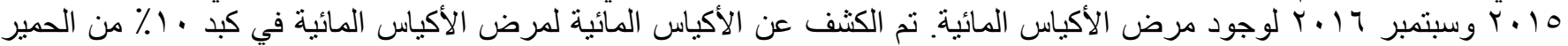

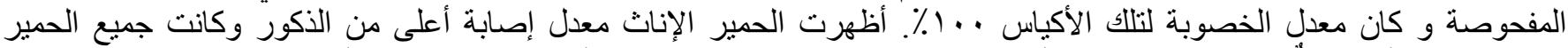

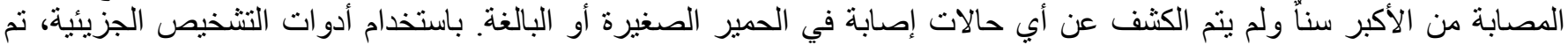

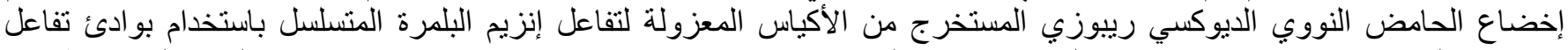

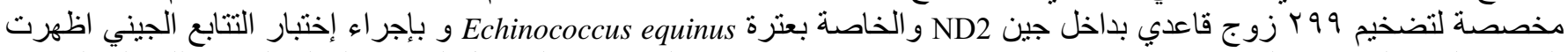

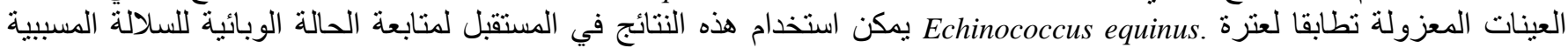

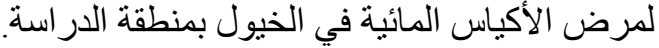

\section{Introduction}

Hydatidosis is an important world-wide zoonotic disease caused by the larval stages (metacestodes) of tapeworm parasites of the genus Echinococcus. These cestodes have an indirect, life cycle, with carnivores (canids) as definitive hosts and herbivorous or omnivorous mammals as intermediate hosts. Adult tapeworms inhabit 
the small intestine of the definitive host and these release gravid segments containing eggs into the environment via feces. When these eggs are ingested by intermediate hosts, the latter become infected with larvae of Echinococcus spp., fluid-filled hydatid cysts (HC) formed mainly in their livers and lungs, in which protoscoleces are produced as the next generation of tapeworms. Their growth can cause severe illness and death in the intermediate host (1). Infection with E.granulosus in intermediate hosts is typically asymptomatic, although there have been a few cases noted of long-standing, heavy infections (2).

The disease is endemic in many parts of the world. It is one of the major zoonotic parasitic diseases in the Middle East and Arab North Africa, from Morocco to Egypt (3). In Egypt, existing studies which have been carried out to determine the infection rate of $\mathrm{HC}$ among Egyptian equids showed that it was $4.62 \%$ in Qaliobia and Giza Governorates (4), 6.89\% in Beni-Suef Governorate (5) and $14.2 \%$ in Giza Governorate (3). More widely, the infection rate of equids with $\mathrm{HC}$ in different countries was found to be $16.7 \%$ in Morocco (6), $0.27 \%$ in Italy (7), $8.9 \%$ in Chile (8), $12.2 \%$ in Romania (9) and $2 \%$ in Iran (10).

Evidence is being accumulated that genetic heterogeneity is common within the species of the genus Echinococcus. Thus, it may be said that a strain of Echinococcus can be defined as a group of individuals which differs statistically from other groups of the same species in gene frequencies. This has a significant impact on the epidemiology, pathogenicity and control of hydatid disease (11).

For identification of E.granulosus at the species level, the use of morphological and biological studies have provided extremely useful information (10) but it should be considered that these features are variable. They may be influenced by host and environmental factors and may not necessarily reflect distinctness at the genetic level. Molecular techniques allow a direct characterization of the genome of the parasite and these techniques have the advantage that they are not confounded by variability induced by the host or the environment $(11,12)$.

Molecular genetic studies based on mitochondrial DNA analysis have demonstrated that E. granulosus is actually a complex of genotypes that exhibit a marked genetic variability. At least ten distinct genotypes (G1-G10) have been identified within the E. granulosus complex. These include two sheep strains (G1 and G2), two bovid strains (G3 and G5), a horse strain (G4), a camel strain (G6), two pig strains (G7 and G9) and cervid strains (G8 and G10) $(5,13,14)$. E.equinus (also known as E. granulosus G4 or horse strain) uses equids as specific intermediate hosts $(1,5,7,15-21)$. No human cases of infection with E.equinus are known, and the epidemiological evidence suggests that it may be non-pathogenic to humans $(13,15)$. Other
Echinococcus strains (E. granulosus G1 or sheep strain) might also cause hydatidosis in equids $(7,22)$.

The aim of this study is to genetically characterize the isolates of HC from donkeys examined in Giza zoo, in order to determine the causative strain of hydatidosis of donkeys in the study area.

\section{Material and methods}

\section{Study area}

This study was conducted from October 2015 to September 2016 in the abattoir of the Giza Zoo, Egypt. The animals that were examined were brought to the zoo from the centers and nearby cities of Cairo and Giza Governorates, where they were slaughtered to feed predators. The samples were collected from (40) donkeys of both sexes and of varying ages. Donkeys were grouped into three age groups according to (4) (Table 1).

Table 1: Number of donkeys examined, according to sex and age

\begin{tabular}{lcc}
\hline Age groups & \multicolumn{2}{c}{ Sex } \\
\cline { 2 - 3 } & Males & Females \\
\hline Young donkeys ( $<2$ years) & 2 & 1 \\
Adult donkeys $(\geq 2$ or $\leq 10$ years $)$ & 9 & 8 \\
Old donkeys $(>10$ years) & 7 & 13 \\
\hline Total $(40)$ & 18 & 22 \\
\hline
\end{tabular}

\section{Collection of parasite material}

After slaughtering, each donkey was examined for the presence of $\mathrm{HC}$, using palpation and incision (3). The intact cysts were removed from an infected animal, put in separate, polyethylene bags and labeled with the necessary data (age, sex, infected organ and date of collection), then sent in an ice box to the Biotechnology lab, Animal Health Research Institute, Dokki, for examination. In the lab, each cyst was washed with distilled water to remove debris, incised and then $\mathrm{HC}$ fluid was aspirated using a sterile syringe. The collected fluid was centrifuged at $3000 \mathrm{rpm}$ for 7 minutes and the sediment was collected.

\section{Checking the fertility of cysts}

The sediment of each cyst was observed under a light microscope at 40x magnification. Cysts with no protoscoleces were considered to be sterile cysts (23). After being examined, the sediment was preserved in $70 \%$ ethyl alcohol for further use.

\section{Molecular characterization of $\mathrm{HC}$ isolates Parasite material}

$70 \%$ ethyl alcohol preserved $\mathrm{HC}$ sediments (containing protoscoleces) which were collected from fertile HC. 


\section{DNA extraction}

DNA was extracted from preserved protoscoleces using the Thermo Scientific Gene JET Genomic DNA Purification Kit according to manufacturer's instructions (Cat No \#K0722).

\section{Oligonucleotide primers}

For conventional PCR, oligonucleotide primers were synthesized as follows:- forward:

\section{5'-GGTTTTGAGATA CATAATAATGTCCGGAC-3'} and reverse:

5'-CTCACACCAAGCACCTACACATAAATATAGTT-3' (20). These oligonucleotide primers were constructed to target 299 bp within the NADH (Nicotinamide Adenine Dinucleotide) dehydrogenase subunit 2 (ND2) gene, which is considered specific to the E.equinus genotype.

\section{Polymerase Chain Reaction (PCR) amplification}

DNA amplification was done in $20 \mu \mathrm{L}$ reaction volume containing $4 \mu \mathrm{L} 5 \times$ FIREPol Master Mix, $0.5 \mu \mathrm{L}$ $(10 \mathrm{pmol} / \mu \mathrm{L})$ from each primer, $3 \mu \mathrm{L}$ extracted DNA and then filled up to $20 \mu \mathrm{L}$ with DNAse/RNAse free water. Negative control (no-DNA) reaction was included in PCR amplification. The amplification procedure was carried out using MJ Mini thermal cycler (Bio-Rad) with the following thermal profile: $95^{\circ} \mathrm{C}$ for $5 \mathrm{~min}$ as an initial denaturation, 35 cycles of $94^{\circ} \mathrm{C} / 30 \mathrm{~s}$ (denaturation step), $57^{\circ} \mathrm{C} / 30 \mathrm{~s}$ (annealing step), $72^{\circ} \mathrm{C} / 30 \mathrm{~s}$ (extension step) and a final extension at $72^{\circ} \mathrm{C}$ for 5 min. $5 \mu \mathrm{L}$ aliquots of PCR products were then fractionated on $1.5 \%$ agarose gel stained with ethidium bromide, which marked by $50 \mathrm{bp}$ DNA ladder (Genedirex). The electric current was adjusted at $100 \mathrm{~V}$ for 30 minutes, and then gel was visualized and photographed using the Bio-Rad gel documentation system.

\section{DNA sequencing}

Samples were considered positive for E.equinus when a single band of DNA at 299 bp was evident in ethidium bromide agarose gel. The PCR amplicons $(n=2)$, after being fractionated on ethidium bromide agarose gel, were cut from the gel and purified using the Thermo Scientific Gene JET Gel Extraction Kit (Cat No. K0691). The purified PCR products were then sequenced in one direction, using reverse primer. The sequencing procedure was done at the Genome unit, Animal Health Research Institute, Dokki.

\section{Computer-assisted sequences and phylogenetic analysis}

The electropherogram of each sequence $(n=2)$ was checked by eye. The resulting nucleotide sequences were aligned and compared to each other using BioEdit software (http://www.mbio.ncsu.edu/BioEdit/bioedit.html). Using the Basic Local Alignment Search Tool (BLAST) (http://blast.ncbi.nlm.nih.gov), the DNA sequences obtained were subsequently aligned and compared with verified sequences of E.equinus and other E.granulosus strains available in the Genbank (Table 2).

Table 2: Genbank accession numbers of verified sequences of Echinococcus equinus and other E.granulosus strains used in analysis

\begin{tabular}{cl}
\hline $\begin{array}{c}\text { Accession } \\
\text { numbers }\end{array}$ & Genotype \\
\hline KY766905.1 & Echinococcus equinus isolate G4 mitochondrion partial genome \\
AB786665.1 & Echinococcus equinus mitochondrial DNA complete genome sample code: EEQU/JEN \\
AF346403.1 & Echinococcus equinus mitochondrion complete genome \\
AB235848.1 & Echinococcus canadensis mitochondrial DNA complete genome genotype G8 \\
AB208063.1 & Echinococcus canadensis mitochondrial DNA complete genome \\
AB745463.1 & Echinococcus canadensis mitochondrial DNA complete genome genotype: G10 \\
AB786664.1 & Echinococcus granulosus mitochondrial DNA complete genome sample code: 52LI07 \\
AB208546.1 & Echinococcus vogeli mitochondrial DNA complete genome \\
AF297617.1 & Echinococcus granulosus genotype 1 mitochondrion complete genome \\
AB208064.1 & Echinococcus shiquicus mitochondrial DNA complete genome \\
KC897684.1 & Echinococcus granulosus isolate P NADH dehydrogenase subunit 2 (ND2) gene complete cds mitochondrial \\
KC897682.1 & Echinococcus granulosus isolate N NADH dehydrogenase subunit 2 (ND2) gene complete cds mitochondrial \\
KU601616.1 & Echinococcus granulosus genotype Omo mitochondrion complete genome \\
KY766901.1 & Echinococcus granulosus isolate TUR1 mitochondrion partial genome \\
KY766900.1 & Echinococcus granulosus isolate SPA1 mitochondrion partial genome \\
KY766894.1 & Echinococcus granulosus isolate IRA1 mitochondrion partial genome \\
AB235846.1 & Echinococcus ortleppi mitochondrial DNA complete genome genotype G5 \\
AY684274.1 & Taenia saginata mitochondrion complete genome \\
\hline
\end{tabular}


The phylogenetic tree was constructed by the software program Mega 7 (http://www.megasoftware.net), using the neighbor-joining method. Taenia saginata (AB684274.1) was used as an out group. The sequences analyzed in the present study were then deposited in the Genbank under accession numbers (MF442262 and MF442263).

\section{Results}

Out of 40 examined donkeys, 4 donkeys harbored HC. The infection rate was $10 \%$. Female donkeys were harbored $\mathrm{HC}$ more than males (Table 3). All infected donkeys were old aged with no cases of infection were detected in young or adult donkeys. In all infected animals, the cysts were present only in the liver and there was no evidence of cyst formation in the lungs. The number of cysts observed in infected livers ranged from 2 to 4 cysts, and these were of different sizes. All the collected cysts were fertile and harbored protoscolices (Table 4).

On using extracted DNA as a template for PCR amplification, using synthesized oligonucleotide primers, the result showed $299 \mathrm{bp}$ fragments in ethidium bromide agarose gel which were specific for E.equinus (Fig 1).

The alignment of partial nucleotide sequence of ND2 isolates obtained from examined donkeys showed that they had $100 \%, 90 \%, 90 \%, 89 \%$ and $92 \%$ homology to E.equinus (AB786665.1), E.granulosus (KJ559023.1) E.canadensis (AB208063.1), E.vogeli (AB208546.1) and E.shiquicus (AB20864.1) respectively. When the nucleotide sequences of isolates were aligned to the reference nucleotide sequence of E.equinus accessed in the Genbank they revealed insertion and deletion of nucleotides at several positions (Fig 2). The phylogenetic analysis showed that the obtained isolates belonged to E.equinus (Fig 3).

Table 3: Infection rate of $\mathrm{HC}$ in the examined donkeys

\begin{tabular}{lcc}
\hline Sex & $\begin{array}{c}\text { Number of the } \\
\text { examined animals }\end{array}$ & $\begin{array}{c}\text { Number of the } \\
\text { infected animals }\end{array}$ \\
\hline Males & 18 & 1 \\
Females & 22 & 3 \\
\hline Total & 40 & 4 \\
\hline Infection rate (\%) & \multicolumn{2}{c}{$10 \%$} \\
\hline
\end{tabular}

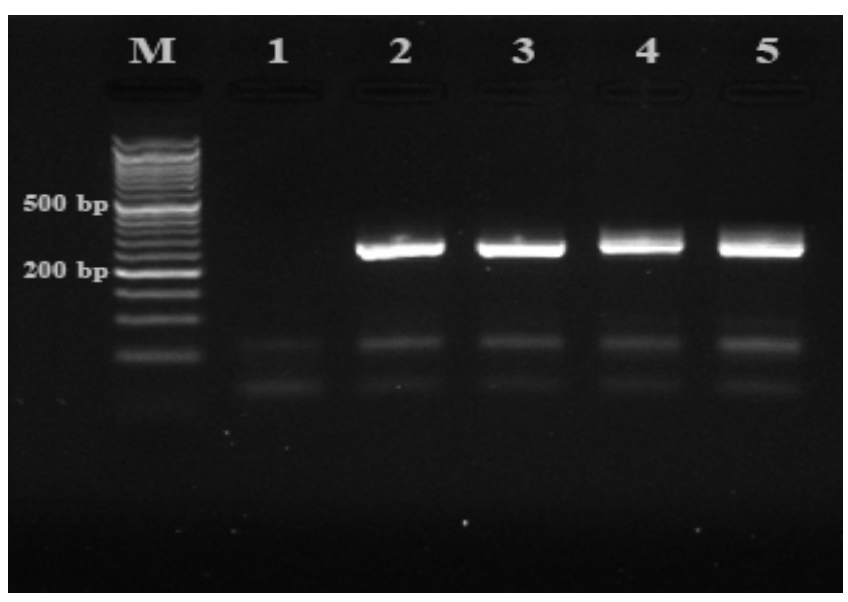

Figure 1: $1.5 \%$ ethidium bromide stained agarose gel electrophoresis. Lane M: 50 bp ladder marker. Lane 1: negative control. Lane 2-5: amplified fragments of $299 \mathrm{bp}$ resulted from amplification of extracted DNA using synthesized oligonucleotide primers specific for E.equinus.

\section{Discussion}

In the present study, the infection rate of $\mathrm{HC}$ is $(10 \%)$. This result is considered higher than results obtained by $(4,5)$ while it is lower than the result obtained by $(3)$, who carried out their necropsy examination in different localities in Egypt. The infection rate is higher than that obtained by $(7,8,10)$ while it is lower than that obtained by $(6,9)$ who carried out their studies in different parts of the world. The difference in infection rate may be due to variation in environmental factors such as temperature, humidity and the nature of the pasture (24).

All infected animals harbored HC only in their livers, a result that agrees with that obtained by $(4,10)$ while it disagrees with results obtained by (5-8) who showed presence of $\mathrm{HC}$ in the liver and lung of infected animals, and (1) in Germany, who demonstrated the presence of $\mathrm{HC}$ only in the lung of infected mares. This may be due to the bile duct in the liver, which receives blood with oncospheres after it passes the duodenum (24).

Table 4: Number, distribution and fertility of $\mathrm{HC}$ in infected donkeys

\begin{tabular}{|c|c|c|c|c|c|c|c|c|c|c|}
\hline \multirow{3}{*}{ Donkey } & \multirow{3}{*}{ Age } & \multirow{3}{*}{ Sex } & \multicolumn{8}{|c|}{ Recovered hydatid cysts (HC) } \\
\hline & & & \multirow{2}{*}{ No. of cysts } & \multirow{2}{*}{ Inf. organ } & \multicolumn{2}{|c|}{ Fertile } & \multicolumn{2}{|c|}{ Sterile } & \multicolumn{2}{|c|}{ Calcified } \\
\hline & & & & & No. & $\%$ & No. & $\%$ & No. & $\%$ \\
\hline 1 & 18 year & Female & 2 & Liver & 2 & $100 \%$ & - & - & - & - \\
\hline 2 & 12 year & Female & 3 & Liver & 3 & $100 \%$ & - & - & - & - \\
\hline 3 & 28 year & Female & 2 & Liver & 2 & $100 \%$ & - & - & - & - \\
\hline 4 & 11 year & Male & 4 & Liver & 4 & $100 \%$ & - & - & - & - \\
\hline
\end{tabular}



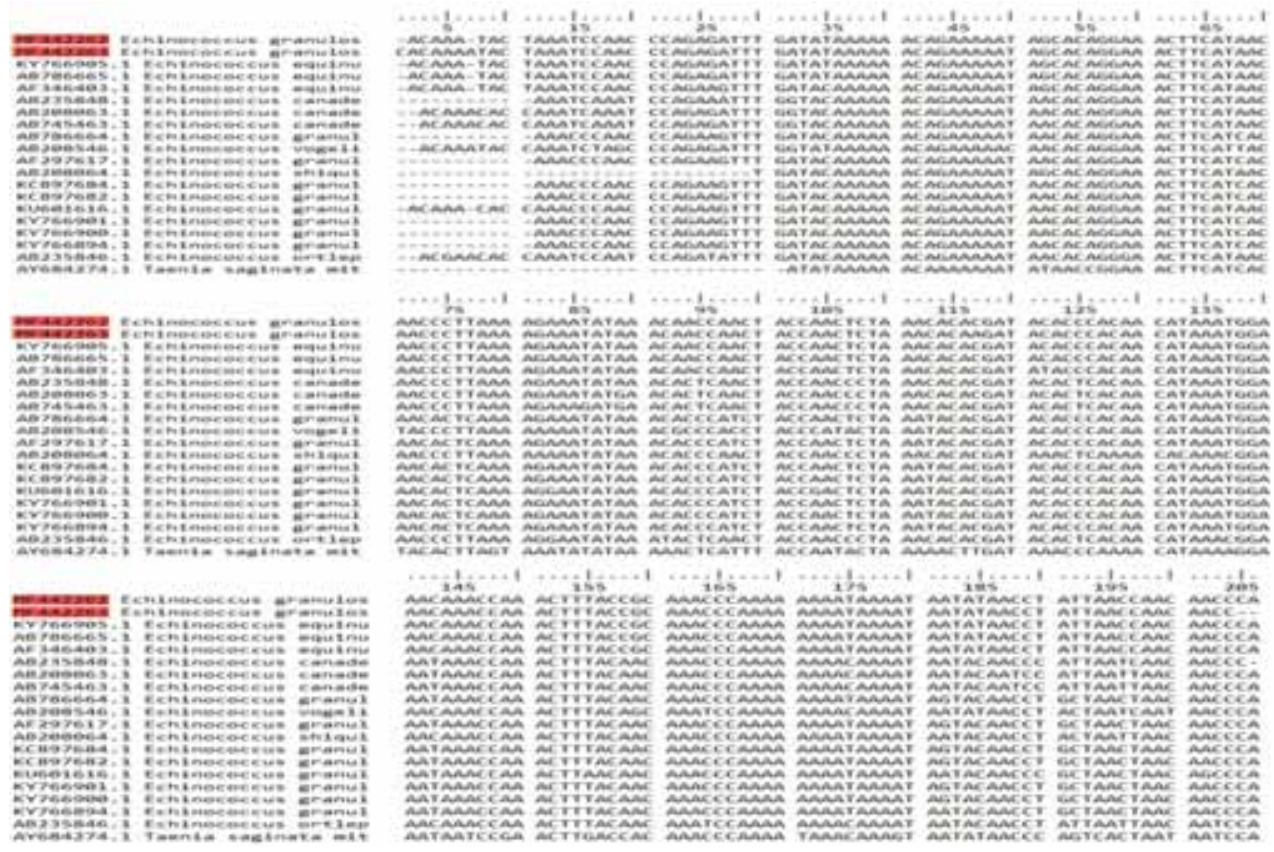

Figure 2: Alignment of nucleotide sequence of E.equinus of isolates obtained from donkeys examined at Giza Zoo, Egypt (labeled) with reference to sequences of E.equinus accessed in the genbank, in addition to sequences of different genotypes of genus Echinococcus.

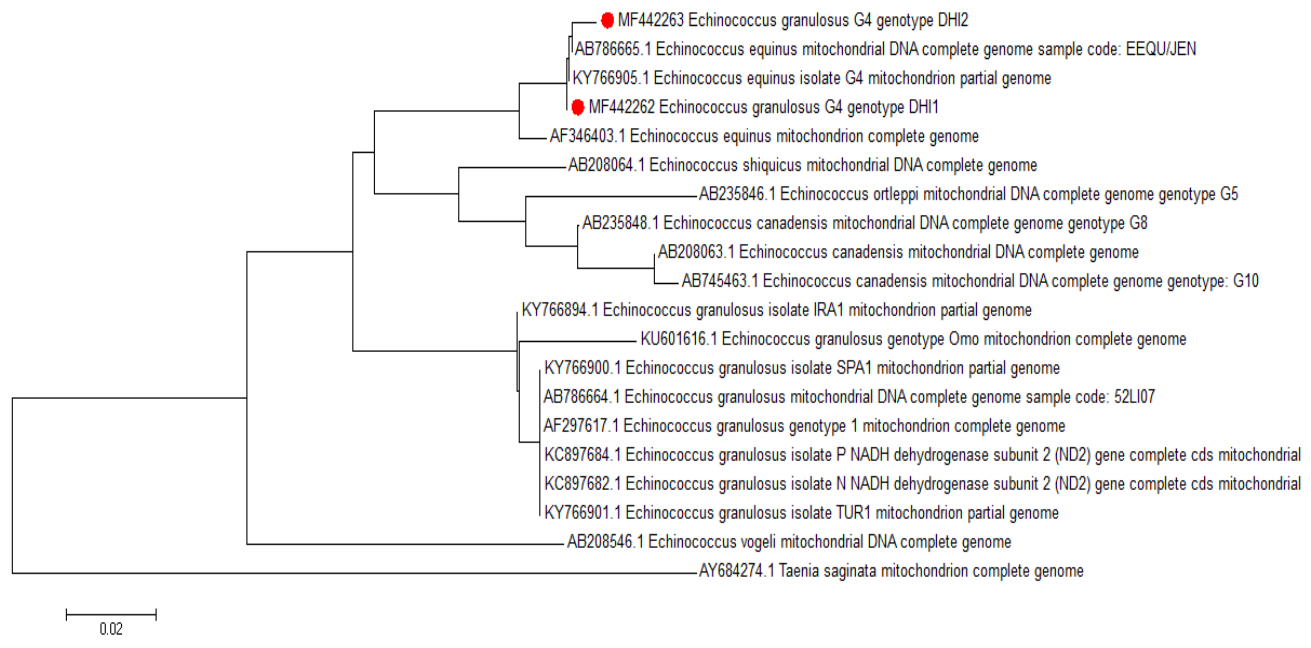

Figure 3: Genetic relationship of E.equinus isolates obtained from examined donkeys at Giza Zoo, Egypt (labeled) with reference to sequences of E.equinus accessed in the genbank and with reference to sequences of other genotypes of genus Echinococcus which were constructed by phylogenetic analysis of the nucleotide sequence of the ND2 gene.

The livers infected with $\mathrm{HC}$ in this study show a fertility rate $(100 \%)$ which agrees with the result obtained by (10) and disagrees with the result obtained by $(3,5,7)$. This may be because the causative strain (E.equinus) tends to produce very well-developed hydatid cysts (7).

The prevalence of $\mathrm{HC}$ according to sex showed that most cases of infection occurred in female donkeys; only one case was diagnosed in male donkeys which agrees with the result obtained by $(4,25)$. (26) Proved that infection with $\mathrm{HC}$ is equally prevalent in male and female donkeys. This may be because female donkeys have a long gestation period, and because of the adverse environmental conditions suffered by donkeys in the Middle East countries (25). 
Our result showed further that the infections with $\mathrm{HC}$ occurred in older animals, and this disagrees with the results obtained by (4), who reported that $\mathrm{HC}$ infection occurs preferentially in adult donkeys. The result agrees with those obtained by $(25,26)$ who reported that rate of infection increases in older animals. This may be because older donkeys have a higher rate of exposure to infective stages (27)

The PCR amplification of the ND2 gene produced bands of expected length (299bp) which agrees with the results obtained in previous study (20). Sequencing of amplified products showed the homology of these isolates to the reference sequence of E.equinus accessed in the Genbank which confirmed that equids are the specific intermediate host for E.equinus (1,15-21). On the other hand, $(7,22)$ have reported that horses can be infected with E.granulosus s.s. (sheep strain) as well as by E.equinus (horse strain). Our results agreed with that obtained by (5), who confirmed that Egyptian donkeys are the main host for E.equinus with up to the present time no incidence of infection with other genotypes.

As the zoo's abattoir represents the main location for the slaughtering of donkeys for dietary consumption (feeding of the zoo's predator animals), we recommend the strict post mortem inspection of slaughtered donkeys, prior to introducing them as food, as well as the hygienic disposal of organs infected with HC. This will result in the breaking of the life cycle between the intermediate and the final host, with a subsequent decreasing the frequency of this disease. Furthermore, studies on hydatidosis disease in farm animals should be continued to elucidate the role of these animals in transmission of such serious disease to human populations.

\section{Acknowledgment}

The authors gratefully acknowledge the assistance of all the staff workers at the Giza Zoo, Egypt, for their appreciable help and for their permission to examine the carcasses of the slaughtered donkeys in the zoo's abattoir. Grateful thanks to Dr/ Ahmed Anwar Wahba (Professor of Parasitology, Parasitology department, Animal Health Research Institute, Dokki, Egypt) for his valuable advices.

\section{References}

1. Blutke A, Hamel D, Huttner M, Gehlen H, Romig T, Pfister K, Hermanns W. Cystic echinococcosis due to Echinococcus equinus in a horse from southern Germany. $\mathrm{J}$ Vet diagnostic Investig. 2010;22:458-462.

2. Daryani A, R.Alaei, R.Arab, M S, H.Dehghan, H.Ziaei. Prevalence of Hydatid Cyst in Slaughtered Animals in Northwest Iran. J Anim Vet Adv. 2006;5(4):330-334.

3. Mahdy OA, Abdel Maogood SZ, Abdel Wahab AM, El-Bahy MM. Epidemiological and molecular characterization of antigens extracted from Hydatid cysts of camel, cattle and donkeys in Egypt. Int J Basic Appl Sci. 2014;3(2):93-98.
4. Radwan AMM. An investigation on parasitic infection in equines. Banha Univ Vet Med Dep Parasitol (MVSC). 2009.

5. Aboelhadid SM, El-Dakhly KM, Yanai T, Fukushi H, Hassanin KM. Molecular characterization of Echinococcus granulosus in Egyptian donkeys. Vet Parasitol. 2013;193:292-296.

6. Azlaf R, Dakkak A. Epidemiological study of the cystic echinococcosis in Morocco. Vet Parasitol. 2006;137:83-93.

7. Varcasia A, Garippa G, Pipia AP, Scala A, Brianti E, Giannetto S, Battelli G, Poglayen G, Micagni G.. Cystic echinococcosis in equids in Italy. Parasitol Res. 2008;102:815-818.

8. Acosta-Jamett G, Cleaveland S, Cunningham AA, Bronsvoort BM de C, Craig PS. Echinococcus granulosus infection in humans and livestock in the Coquimbo region, north-central Chile. Vet Parasitol. 2010;169:102-110.

9. Neghina R, Neghina AM, Marincu I, Iacobiciu I. Epidemiology and epizootology of cystic echinococcosis in Romania 1862-2007. Foodborne Pathog Dis. 2010;7(6):613-618.

10. Eslami A, Shayan P, Bokaei S. Morphological and genetic characteristics of the liver hydatid cyst of a donkey with Iran origin. Iran J Parasitol. 2014;9(3):302-310.

11. Taha HA. Genetic variations among Echinococcus granulosus isolates in Egypt using RAPD-PCR. Parasitol Res. 2012;111:1993-2000.

12. Bowles J, McManus DP. Molecular variation in Echinococcus. Acta Trop. 1993;53:291-305.

13. Thompson RCA, McManus DP. Towards a taxonomic revision of the genus Echinococcus. Trends Parasitol. 2002;18(10):452-457.

14. Cardona GA, Carmena D. A review of the global prevalence, molecular epidemiology and economics of cystic echinococcosis in production animals. Vet Parasitol. 2013;192:10-32.

15. Jenkins DJ, Romig T, Thompson RCA. Emergence/re-emergence of Echinococcus spp. - A global update. Int J Parasitol. 2005;35:120519.

16. Romig T, Dinkel A, Mackenstedt U. The present situation of echinococcosis in Europe. Parasitol Int. 2006;55:187-191.

17. Nakao M, McManus DP, Schantz PM, Craig PS, Ito A. A molecular phylogeny of the genus Echinococcus inferred from complete mitochondrial genomes. Parasitology. 2007;134:713-722.

18. Thompson RCA. The taxonomy, phylogeny and transmission of Echinococcus. Exp Parasitol. 2008;119:439-446.

19. Saarma U, Jõgisalu I, Moks E, Varcasia A, Lavikainen A, Oksanen A, Simsek S, Andresiuk, V, Denegri G, González LM, Ferrer E, Gárate T, Rinaldi L, Maravilla P. A novel phylogeny for the genus Echinococcus, based on nuclear data, challenges relationships based on mitochondrial evidence. Parasitology. 2009;136:317-328.

20. Lett WS. Detection of Echinococcus granulosus and Echinococcus equinus in Dogs and Epidemiology of Canine Echinococcosis in the UK. Univ. Salford,School Environ. Life Sci. 2013.

21. Simsek S, Cevik A. First detection and molecular characterization of Echinococcus equinus in a mule in Turkey. Acta Parasitol. 2014;59(4):773-777.

22. Utuk AE, Simsek S. Molecular characterization of the horse isolate of Echinococcus granulosus in Turkey. J Helminthol. 2013;87:305-308.

23. Shahnazi M, Jafari A, Javadi M, Saraei M. Fertility of Hydatid Cysts and Viability of Protoscoleces in Slaughtered Animals in Qazvin, Iran. J Agric Sci. 2013;5(1):141-146.

24. Elmajdoub LO, Rahman WA. Prevalence of Hydatid Cysts in Slaughtered Animals from Different Areas of Libya. Open J Vet Med. 2015;5:1-10

25. Abo-shehada MN. Prevalence of Hydatidosis in Donkeys from Central Jordan. Vet Parasitol. 1988;30:125-130.

26. Mukbel RM, Torgerson PR, Abo-Shehada MN. Prevalence of hydatidosis among donkeys in northern Jordan. Vet Parasitol. 2000;88:35-42

27. Ibrahim MM. Study of cystic echinococcosis in slaughtered animals in Al Baha region, Saudi Arabia: Interaction between some biotic and abiotic factors. Acta Trop. 2010;113:26-33. 